\title{
BMJ Open Effectiveness of implementation strategies in improving physician adherence to guideline recommendations in heart failure: a systematic review protocol
}

Harriette G C Van Spall, ${ }^{1,2,3}$ Deepti Shanbhag, ${ }^{4}$ Itzhak Gabizon, ${ }^{1}$ Quazi Ibrahim, ${ }^{2}$ Ian D Graham, ${ }^{5}$ Karen Harlos, ${ }^{6}$ R Brian Haynes, ${ }^{3}$ Stuart J Connolly ${ }^{1,2,3}$

To cite: Van Spall HGC, Shanbhag D, Gabizon I, et al. Effectiveness of implementation strategies in improving physician adherence to guideline recommendations in heart failure: a systematic review protocol. BMJ Open 2016;6: e009364. doi:10.1136/ bmjopen-2015-009364

- Prepublication history and additional material is available. To view please visit the journal (http://dx.doi.org/ 10.1136/bmjopen-2015009364).

Received 10 July 2015 Revised 18 February 2016 Accepted 3 March 2016

CrossMark

For numbered affiliations see end of article.

Correspondence to Dr Harriette GC Van Spall; Harriette.VanSpall@phri.ca

\section{ABSTRACT}

Introduction: The uptake of Clinical Practice Guideline (CPG) recommendations that improve outcomes in heart failure (HF) remains suboptimal. We will conduct a systematic review to identify implementation strategies that improve physician adherence to class I recommendations, those with clear evidence that benefits outweigh the risks. We will use American, Canadian and European HF guidelines as our reference. Methods and analysis: We will conduct a literature search in the databases of MEDLINE, EMBASE, HEALTHSTAR, CINAHL, Cochrane Library, Campbell Collaboration, Joanna Briggs Institute Evidence Based Practice, Centre for Reviews and Dissemination and Evidence Based Practice Centres. We will include prospective studies evaluating implementation interventions aimed at improving uptake of class I CPG recommendations in HF. We will extract data in duplicate. We will classify interventions according to their level of application (ie, provider, organisation, systems level) and common underlying characteristics (eg, education, decision-support, financial incentives) using the Cochrane Effective Practice and Organisation of Care Taxonomy. We will assess the impact of the intervention on adherence to the CPGs. Outcomes will include proportion of eligible patients who were: prescribed a CPG-recommended pharmacological treatment; referred for device consideration; provided self-care education at discharge; and provided left ventricular function assessment. We will include clinical outcomes such as hospitalisations, readmissions and mortality, if data is available. We will identify the common elements of successful and failing interventions, and examine the context in which they were applied, using the Process Redesign contextual framework. We will synthesise the results narratively and, if appropriate, will pool results for meta-analysis.

Discussion and dissemination: In this review, we will assess the impact of implementation strategies and contextual factors on physician adherence to HF CPGs. We will explore why some interventions may succeed in one setting and fail in another. We will disseminate our findings through briefing reports, publications and presentations.

\section{Strengths and limitations of this study}

- Our study will compare the effectiveness of implementation interventions intended to increase adherence to heart failure (HF) Clinical Practice Guidelines. We will focus on class I recommendations, the benefits of which are strongly supported by evidence.

- We will assess the role of contextual factors in influencing the effectiveness of implementations and will provide context-specific recommendations, where possible.

- Our results will inform implementation strategies at the policy, organisation and provider level to increase evidence-informed care and improve outcomes in HF. While the focus of the study is $\mathrm{HF}$, findings may be generalisable to other complex, chronic health conditions.

- We anticipate that the major limitation of this review will be the study design of the primary studies.

Trial registration number: CRD42015017155.

\section{INTRODUCTION}

Heart failure (HF) is a common condition that burdens patients and the healthcare system. With a prevalence of approximately $10 \%$ in the elderly, it accounts for $1-2 \%$ of the healthcare expenditures in developed countries. ${ }^{1}$ Patients diagnosed with HF face a 1-year mortality risk of nearly $30 \%$. For those hospitalised with the condition, the risk of mortality is substantially higher. ${ }^{1}$

The use of evidence-based therapies, pharmacological and non-pharmacological, have the potential to improve clinical outcomes in $\mathrm{HF}$, and the recommendations 
surrounding their use are published in HF Clinical Practice Guidelines (CPGs). Several organisations, such as the American Heart Association, the Canadian Cardiovascular Society, and the European Society of Cardiology publish and update HF guidelines. ${ }^{2-4}$ On the basis of the strength, depth and breadth of the evidence, current class I/level A recommendations for patients with $\mathrm{HF}$ and reduced left ventricular (LV) systolic function include prescription of pharmacological treatments such as $\beta$-blockers, ACE inhibitors (ACEIs), angiotensin receptor blockers (ARBs), and aldosterone antagonists. Class I recommendations also include the use of devices such as the implantable cardioverter defibrillator (ICD), and cardiac resynchronisation therapy (CRT), and services such as self-care education. ${ }^{2}$ Implementation of guideline class I recommendations is associated with a reduction in hospitalisation rates. ${ }^{5}$

Despite the existence of CPGs, the uptake of recommendations into routine clinical practice remains slow and inconsistent. A recent study estimated that optimal implementation of guideline recommendations in $\mathrm{HF}$ could prevent 67996 deaths a year in the USA alone. ${ }^{6}$ To bridge the gap between evidence and practice, efforts must be directed toward implementing strategies that can narrow these gaps. ${ }^{7}$ Several studies have assessed interventions that could facilitate the uptake of evidence-based recommendations. Provider-level interventions that have been examined include audit and feedback, ${ }^{8}$ computer-assisted clinical decision support, ${ }^{9}$ educational materials, and continuing education meetings. ${ }^{8}$ Organisational/systems-level interventions that have been studied include clinical pathways, ${ }^{10}$ nurse-led $\mathrm{HF}$ management, ${ }^{11}$ and specialised $\mathrm{HF}$ clinics. ${ }^{12}$ Interventions that address factors within the broader socioeconomic context include financial incentives such as fee-for-performance or quality-based procedures. ${ }^{13}$

A pre-requisite to implementing strategies to improve clinical care is understanding which ones are successful. Delivering evidence-informed care in $\mathrm{HF}$ has unique challenges as the typical patient with HF receives care from multiple providers across multiple care settings; interventions to improve care may need to target not only providers but also systems, and the success of the interventions may depend on contextual factors, such as the characteristics of the organisation or health system. While previous reviews have assessed the effectiveness of interventions that improve quality of care, ${ }^{14}$ none, to our knowledge, have explored this subject within the area of $\mathrm{HF}$, and none have analysed the contextual factors that influence the success of an implementation strategy.

Our study is guided by an Applied Health Research Question (AHRQ) - a question posed by a health system policy or decision-maker to guide planning, policy and programme development. ${ }^{15}$ The primary objective of this systematic review is to synthesise and evaluate the effectiveness of implementation interventions in increasing adherence to HF CPGs. Outcomes will include the proportion of eligible patients who were: prescribed a guideline-recommended pharmacological treatment; referred for ICD or CRT; provided self-care education at discharge; and received LV function assessment. If clinical outcomes such as hospitalisation, readmissions, or mortality rates are reported in the primary studies, we will evaluate the effectiveness of implementation interventions in improving these. A secondary objective is to identify the contextual factors associated with success or failure of the intervention.

\section{METHODS AND ANALYSIS \\ Eligibility criteria}

We have developed eligibility criteria from the research question: What implementation interventions increase physician adherence to heart failure Clinical Practice Guidelines, and how effective are these interventions relative to usual care? We framed our criteria according to the following PICOS (Population, Intervention, Comparator, Outcomes, Study design) elements:

Population: We will include only studies with patients who are treated for $\mathrm{HF}$, using the diagnosis of $\mathrm{HF}$ as defined in the included studies.

Intervention: We will include trials evaluating one or more interventions aimed at improving physician adherence to class I guideline-based treatment of $\mathrm{HF}$, and will categorise them according to the level of the intervention (eg, provider-level, organisation-level, systems-level), and on the basis of common underlying characteristics (eg, education, decision-support, financial incentives) according to the Cochrane Effective Practice and Organization of Care (EPOC) Taxonomy. ${ }^{16}$

Comparator: We will include studies that use standard or usual care as a comparator strategy.

Outcomes: Our primary outcomes will be: proportion of eligible patients prescribed a guideline-recommended pharmacological treatment such as $\beta$-blockers, ACEIs, ARBs and aldosterone antagonists; proportion of patients prescribed an exercise regimen; proportion of patients referred for ICD or CRT consideration; proportion of patients provided self-care education at discharge and proportion of patients provided LV function assessment. Among interventions aiming to improve pharmacological treatment, we will also assess the proportion of patients titrated to the guideline-recommended target dose of $\beta$-blocker, ACEI, ARB or aldosterone antagonist. The outcomes used to measure physician adherence to HF guidelines in each study will be consistent with guideline recommendations that were contemporary to the time at which the study was conducted.

Secondary outcomes will include clinical outcomes, such as HF-related hospitalisations, readmissions and mortality.

Study design: We will include randomised controlled trials (RCTs; including cluster RCTs), cohort studies (with comparisons), interrupted time series (ITS) studies and controlled before-after studies. We will exclude case-series studies, reviews, editorials, letters to 
the editor, non-English studies and studies published prior to 1990.

\section{Information sources}

We will conduct a systematic search of the literature, restricted to the English language for articles that were published from 1990 to the present in MEDLINE, EMBASE, HEALTHSTAR, CINAHL, The Cochrane Library, The Campbell Collaboration, The Joanna Briggs Institute Evidence Based Practice Database, The AHRQ Evidence-based Practice Centers' Research Reports, and the University of York Centre for Reviews and Dissemination Database.

\section{Search strategy}

We will develop our main strategy, with the guidance of an experienced information specialist, in MEDLINE (Ovid). Our preliminary search strategy includes the terms: heart failure, guideline adherence, practice guideline, evidence-based medicine, implement. (The preliminary search strategy for MEDLINE is available in online supplementary appendix 1.)

We will apply the preliminary MEDLINE search strategy (see online supplementary appendix1) in the other databases. Our secondary search will include each of the different intervention types and heart failure (see online supplementary appendix2). We will search for additional articles in the reference lists of reviews and editorials identified through the two search strategies.

An exploratory search produced approximately 4400 results. After removing duplicates and screening articles against our eligibility criteria, we identified approximately 150 articles for full-text review. We anticipate the inclusion of at least 30 primary studies in this review.

\section{Study selection}

We will identify and exclude duplicate reports retrieved from our literature searches. Two authors will independently screen titles and abstracts of the articles to determine eligibility for further consideration. We will resolve disagreements by discussion. Two authors will then independently evaluate the full text of articles selected during preliminary screening according to the eligibility criteria, and record reasons for exclusion of ineligible studies. Disagreements will be resolved by discussion and, when required, by consulting a third author.

\section{Data extraction and management}

We will independently extract the following details in duplicate: methods (study design, unit of allocation, number of units of allocation, unit of analysis, power calculation, quality criteria, duration of postintervention follow-up, outcome measurement and study setting), characteristics of HF patients (number, clinical diagnosis, New York Heart Association (NYHA) class, comorbidities, age and gender), intervention (targeted behaviour, nature of desired change, medium employed and comparison), contextual factors that may have influenced success of implementation, and outcomes (primary and secondary outcomes and time points reported). Contextual factors, as outlined in the Process Redesign contextual framework, will include the nature of the clinical setting (eg, inpatient, outpatient, community-based care, academic status), the specific roles and responsibilities of the professionals involved in the intervention, the strategies used by an organisation to promote use of the adopted intervention, and the barriers and facilitators of implementation. ${ }^{17}$ We will also evaluate the influence of the broader economic, political and social landscapes within which an organisation exists. ${ }^{17}$ In cases where we find that the description for the implementation strategy is not described in sufficient detail, we will contact the original authors for further clarification.

We will conduct calibration exercises before starting the data extraction, to ensure agreement between the data extractors. This will involve data extractions and quality assessments on a sample of three studies by the data extractors and the lead author (HV). This will be followed by comparison and discussion to ensure that all authors are reporting the same information for each criterion in the data extraction form. We will use results from an intention-to-treat analysis. In the case that effect sizes cannot be calculated with available data, we will contact the original authors to obtain additional data. Where this is not possible, we will carry out the analysis using the available data and assess the impact of study exclusion by conducting a sensitivity analysis.

Two authors will independently extract data from included studies. We will resolve disagreements by discussion and, when required, by consulting the lead author.

\section{Assessment of risk of bias in included studies}

Two authors will independently assess the quality of included studies using the criteria outlined in the EPOC reviews and the Cochrane Handbook for Systematic Reviews of Interventions. ${ }^{18}$ Any disagreement will be resolved by discussion and, when required, by consulting a third author.

The criteria for assessing risk of bias will vary by type of study. RCTs, non-RCTs, and controlled before and after studies will be assessed for random sequence generation, allocation concealment, similar baseline outcome measurements, similar baseline characteristics, adequate reporting of incomplete outcome data, blinded outcome evaluation, and for protection against contamination or selective outcome reporting. Quality assessment of cluster RCTs will additionally include evaluation for recruitment bias, baseline imbalance, loss of cluster and incorrect analysis. In addition, we will use the CONSORT statement to assess the quality and transparency of reporting in RCTs and cluster RCTs. ${ }^{19} 20$

ITS studies will be assessed for protection against secular changes, appropriateness of data analysis, disclosure of reason for the number of points preintervention and postintervention, description of shape of the 
intervention effect, protection against detection bias, completeness of data, reliability of the primary outcome measure, risk of selective outcome reporting, and other risk of bias.

We will summarise the risk of bias within and across studies.

\section{Assessment of reporting biases}

To assess the possibility of reporting biases in our systematic review, we will plot the effect size observed in each trial against the inverse of its $\mathrm{SE}$, to generate a funnel plot. The symmetry of the plot will be assessed visually and formally using the Egger's test.

\section{Confidence in cumulative evidence}

We will evaluate the quality of evidence for all outcomes using the Grading of Recommendations Assessment, Development and Evaluation working group methodology across the domains of risk of bias, consistency, directness, precision and publication bias. ${ }^{21}$ We will rate quality as high (further research is very unlikely to change our confidence in the estimate of effect), moderate (further research is likely to have an important impact on our confidence in the estimate of effect, and may change the estimate), low (further research is very likely to have an important impact on our confidence in the estimate of effect, and is likely to change the estimate) or very low (very uncertain about the estimate of effect).

\section{Data synthesis}

We will classify the implementation strategies according to level of intervention (professional, financial, organisational and regulatory) and the type of intervention (eg, education, decision-support, financial incentives) using EPOC Taxonomy. ${ }^{16}$ We will pool the results within each category in a meta-analysis if the studies are sufficiently similar with respect to study design, setting and treatments for pooling to be meaningful. For studies included in the meta-analyses, an additional table will present the statistical estimates (ORs or relative risk for dichotomous variables, and mean differences for continuous variables, along with $95 \%$ CIs). Among these studies, we will first assess clinical heterogeneity at face value on the basis of included populations, intervention types and outcome measures. If heterogeneity is unclear from this initial assessment, we will use the $\mathrm{I}^{2}$ statistic, defining substantial heterogeneity as $\mathrm{I}^{2}>75 \%{ }^{22}$ For outcomes that do not show substantial heterogeneity, meta-analyses will be performed using Cochrane Collaboration's Review Manager (RevMan) V.5.3.4. The pooled data will be computed under a random-effect model (some degree of heterogeneity is likely), using the Mantel-Haenszel method, and the 95\% CI will be estimated. Results will be displayed as Forest plots. In the case of cluster RCTs in which the unit of allocation is different from the unit of analysis, we will use alternative methods of analysis as outlined in the Cochrane
Handbook for Systematic Reviews of Interventions. ${ }^{18}$ For studies testing two or more implementation strategies, we will use a meta-regression analysis involving mixed models.

Where a meta-analysis is not appropriate, we will narratively synthesise the results. We will apply the Process Redesign contextual framework developed by Rojas Smith et $a .^{17}$ This framework will facilitate an analysis of the relative effectiveness of the implementation interventions on the basis of their inherent characteristics, the inner and outer contexts in which they are applied, the characteristics of the professionals using the intervention, and the process of implementation. The inner context will address factors associated with the clinical setting (eg, inpatient, outpatient, community-based care, academic status). The outer context will refer to characteristics of the broader socioeconomic environment in which the intervention is applied. The characteristics of the professionals will include their roles and responsibilities within their professional teams. Finally, the process of implementation will refer to strategies undertaken to encourage the adoption of the intervention.

\section{Additional analyses}

Prespecified subgroup analyses as defined by type of intervention and practice setting will be performed. We will also perform sensitivity analyses according to type of study design (RCT vs observational) and study size.

\section{Discussion and dissemination}

$\mathrm{HF}$ is a leading cause of healthcare expenditure in developed countries, and suboptimal therapy is associated with hospitalisation and death. ${ }^{6}$ Among chronic conditions, HF consumes a significant proportion of the healthcare budget in most developed countries. ${ }^{1}$ Decision-makers are incentivised to implement strategies that improve outcomes and reduce costs, but strategies to improve HF care are often implemented with limited evaluation of the evidence. The contextual factors that influence the success of an intervention are even less clear.

Through this study, we will determine implementation strategies that improve physician adherence to class I CPGs. We have chosen to focus on class I guidelines, which are supported by clear evidence that benefits outweigh the risks, as their uptake is of greatest relevance to both clinicians and decision-makers. We will assess the contextual factors associated with success of implementation interventions. Exploring the interventions within their contextual frame will help us better understand why some interventions may succeed in one setting but fail in another. For instance, limited resources, time constraints and certain funding models or payment schemes may serve as barriers to change implementation in healthcare organisations, and desired outcomes may not be achieved with these barriers. ${ }^{23}$ Creating a map of successful and failing interventions within a given context will help guide decision-makers and knowledge users in implementing strategies that could improve HF care. 
This is particularly important in an era of escalating healthcare costs, increased usage of hospital-based resources, and gaps in care quality despite best efforts.

While this study focuses on $\mathrm{HF}$ in an effort to answer an AHRQ posed by decision-makers in our jurisdiction, we anticipate that the findings of the review may be generalisable to other chronic illnesses. We will disseminate results via briefing reports, policy papers and publication in peer-reviewed journals. In addition, we will present the results at conferences, Ministry of Health meetings, and hospital committee meetings. The integrated Knowledge Translation approach, ${ }^{24}$ in which decisionmakers partner with researchers on the study, will facilitate quick translation to policy and practice.

\author{
Author affiliations \\ ${ }^{1}$ Department of Medicine, McMaster University, Hamilton, Ontario, Canada \\ ${ }^{2}$ Population Health Research Institute, Hamilton, Ontario, Canada \\ ${ }^{3}$ Department of Clinical Epidemiology and Biostatistics, McMaster University, \\ Hamilton, Ontario, Canada \\ ${ }^{4}$ Faculty of Health Sciences, McMaster University, Hamilton, Ontario, Canada \\ ${ }^{5}$ School of Epidemiology, Public Health and Preventive Medicine, University of \\ Ottawa, Ottawa, Ontario, Canada \\ ${ }^{6}$ Department of Business and Administration, University of Winnipeg, \\ Winnipeg, Manitoba, Canada
}

Acknowledgements The authors thank Intekhab Hossain, who has assisted with the preliminary literature search.

Contributors HGCVS obtained funding, conceived the research question and study design, informed the search strategy and analytic plan, and drafted and edited the manuscript. DS contributed to the search strategy, and drafted and edited the manuscript. IDG contributed to and executed the preliminary search strategy. QI informed the analytic plan and drafted a section of the manuscript. IDG, KH, RBH and SJC contributed to ideas and edited the manuscript for critical content. All authors read and approved the final manuscript.

Funding This research is funded by a grant from the Ontario's Ministry of Health and Long Term Care (MOHLTC). HGCVS receives support from the MOHLTC's Health System Research Fund and Canadian Institutes of Health Research (CIHR). IDG receives support from a CIHR Foundation Grant.

Competing interests None declared.

Provenance and peer review Not commissioned; externally peer reviewed.

Dedication This work is dedicated to the memory of Aubrey Ignatius Van Spall, beloved father and friend.

Open Access This is an Open Access article distributed in accordance with the Creative Commons Attribution Non Commercial (CC BY-NC 4.0) license, which permits others to distribute, remix, adapt, build upon this work noncommercially, and license their derivative works on different terms, provided the original work is properly cited and the use is non-commercial. See: http:// creativecommons.org/licenses/by-nc/4.0/

\section{REFERENCES}

1. Bui AL, Horwich TB, Fonarow GC. Epidemiology and risk profile of heart failure. Nat Rev Cardiol 2011;8:30-41.

2. Yancy CW, Jessup M, Bozkurt B, et al, American College of Cardiology Foundation/American Heart Association Task Force on Practice Guidelines. 2013 ACCF/AHA guideline for the management of heart failure: a report of the American College of Cardiology Foundation/American Heart Association Task Force on practice guidelines. Circulation 2013;128:e240-327.

3. McMurray JJ, Adamopoulos S, Anker SD, et al, ESC Committee for Practice Guidelines. ESC guidelines for the diagnosis and treatment of acute and chronic heart failure 2012: The Task Force for the Diagnosis and Treatment of Acute and Chronic Heart Failure 2012 of the European Society of Cardiology. Developed in collaboration with the Heart Failure Association (HFA) of the ESC. Eur Heart $J$ 2012;33:1787-847.

4. Howlett JG, Chan M, Ezekowitz JA, et al, Canadian Cardiovascular Society Heart Failure Guidelines Panels. The Canadian Cardiovascular Society heart failure companion: bridging guidelines to your practice. Can J Cardiol 2016;32:296-310.

5. Komajda M, Lapuerta P, Hermans N, et al. Adherence to guidelines is a predictor of outcome in chronic heart failure: the MAHLER survey. Eur Heart J 2005;26:1653-9.

6. Fonarow GC, Yancy CW, Hernandez AF, et al. Potential impact of optimal implementation of evidence-based heart failure therapies on mortality. Am Heart J 2011:161:1024-30.

7. Nieuwlaat R, Schwalm JD, Khatib R, et al. Why are we failing to implement effective therapies in cardiovascular disease? Eur Heart $J$ 2013;34:1262-9.

8. Peters-Klimm F, Müller-Tasch T, Remppis A, et al. Improved guideline adherence to pharmacotherapy of chronic systolic heart failure in general practice-results from a cluster-randomized controlled trial of implementation of a clinical practice guideline. J Eval Clin Pract 2008;14:823-9.

9. Braun V, Heintze C, Rufer V, et al. Innovative strategy for implementing chronic heart failure guidelines among family physicians in different healthcare settings in Berlin. Eur J Heart Fail 2011;13:93-9.

10. Fonarow GC, Albert NM, Curtis AB, et al. Improving evidence-based care for heart failure in outpatient cardiology practices: primary results of the Registry to Improve the Use of Evidence-Based Heart Failure Therapies in the Outpatient Setting (IMPROVE HF). Circ Heart Fail 2010;122:585-96.

11. Ansari M, Shlipak MG, Heidenreich PA, et al. Improving guideline adherence: a randomized trial evaluating strategies to increase beta-blocker use in heart failure. Circulation 2003;107:2799-804.

12. Asghar H, Rahko PS. Quality of heart failure management: a comparison of care between a comprehensive heart failure program and a general cardiology practice. Congest Heart Fail 2010;16:65-70.

13. Scott IA, Denaro CP, Bennett CJ, et al. Achieving better in-hospital and after-hospital care of patients with acute cardiac disease. Med $J$ Aust 2004;180(10 Suppl):83-8

14. Grimshaw J, Eccles M, Thomas R, et al. Toward evidence-based quality improvement. Evidence (and its limitations) of the effectiveness of guideline dissemination and implementation strategies 1966-1998. J Gen Intern Med 2006;21(Suppl 2): 14-20.

15. Ontario Ministry of Health and Long-Term Care. Ministry Research Funding Opportunities: Applied Health Research Question. (cited 10 June 2015). http://www.health.gov.on.ca/en/pro/ministry/research/ ahrq.aspx\#guidelines

16. Cochrane Effective Practice and Organisation of Care Review Group. Data Collection Checklist. Cochrane Effective Practice and Organisation of Care. 2002. (cited 15 October 2014). https://epoc. cochrane.org/sites/epoc.cochrane.org/files/uploads/ datacollectionchecklist.pdf

17. Rojas Smith L, Ashok M, Dy SM, et al. Contextual Frameworks for Research on the Implementation of Complex System Interventions. Rockville, MD, USA: Agency for Healthcare and Quality (US), 2014:8-26.

18. Higgins JPT, Green S, ed. Cochrane handbook for systematic reviews of interventions version 5.1.0. (updated March 2011). The Cochrane Collaboration, 2011.

19. Shulz KF, Altman DG, Moher D, et al, CONSORT Group. CONSORT 2010 statement: updated guidelines for reporting parallel group randomised trials. BMJ 2010;340:c332.

20. Campbell MK, Piaggio G, Elbourne DR, et al, CONSORT Group. Consort 2010 statement: extension to cluster randomised trials. BMJ 2012;345:e5661.

21. Balshem $\mathrm{H}$, Helfanda $\mathrm{M}$, Schünemann $\mathrm{HJ}$, et al. GRADE Guidelines: 3. Rating the quality of evidence. J Clin Epidemiol 2011;64:401-6.

22. Higgins JP, Thompson SG, Deeks JJ, et al. Measuring inconsistency in meta-analyses. BMJ 2003;327:557-60.

23. Ellen ME, Léon G, Bouchard G, et al. Barriers, facilitators and views about next steps to implementing supports for evidence-informed decision-making in health systems: a qualitative study. Implement Sci 2014;9:179.

24. Canadian Institute of Health Research. Guide to knowledge translation planning at CIHR: integrated and end-of-grant approaches. Ottawa, ON, Canada: CIHR, 2012:2. 